PROCEEDINGS OF THE

AMERICAN MATHEMATICAL SOCIETY

Volume 128, Number 9, Pages 2593-2596

S 0002-9939(00)05391-0

Article electronically published on February 21, 2000

\title{
BOUNDED VARIATION IN THE MEAN
}

\author{
PAMELA B. PIERCE AND DANIEL WATERMAN \\ (Communicated by Christopher D. Sogge)
}

\begin{abstract}
It is shown that the concept of bounded variation in the mean is not a meaningful generalization of ordinary bounded variation. In fact, it is a characterization of functions which differ from functions of bounded variation on a zero set.
\end{abstract}

Let $f$ be a real-valued function in $L^{1}$ on the circle group $T$. We define the corresponding interval function by $f(I)=f(b)-f(a)$, where $I$ denotes the interval $[a, b]$. Let $0=t_{0}<t_{1}<\cdots<t_{n}=2 \pi$ be a partition of $[0,2 \pi]$, and $I_{k x}=\left[x+t_{k-1}\right.$, $\left.x+t_{k}\right]$. If

$$
V_{m}(f)=\sup \left\{\int_{T} \sum_{k=1}^{n}\left|f\left(I_{k x}\right)\right| d x\right\}<\infty,
$$

where the supremum is taken over all partitions, then $f$ is said to be of bounded variation in the mean (or of bounded variation in the $L^{1}$ norm). We denote the class of all functions which are of bounded variation in the mean by $B V M$. This concept was introduced by Móricz and Siddiqi [MS], who investigated the convergence in the mean of the partial sums of $S[f]$, the Fourier series of $f$.

If $f$ is of bounded variation $(f \in B V)$ with variation $V(f, T)$, then

$$
\int_{T} \sum_{k=1}^{n}\left|f\left(I_{k x}\right)\right| d x \leq 2 \pi V(f, T)
$$

and so it is clear that $B V \subseteq B V M$. Clearly this integral is invariant under an alteration of $f$ on a zero set, and so a function which differs from a $B V$ function on a zero set is in $B V M$.

A straightforward calculation shows that $B V M$ is a Banach space with norm

$$
\|f\|_{B V M}=\|f\|_{1}+V_{m}(f) .
$$

We shall show that bounded variation in the mean implies convergence of $S[f, x]$ to $f(x)$ for every $x$ which is a symmetric Lebesgue point, i.e., for every $f$ which satisfies the symmetric Lebesgue condition,

$$
\frac{1}{h} \int_{0}^{h}|f(x+t)+f(x-t)-2 f(x)| d t=o(1) \quad \text { as } h \rightarrow 0
$$

at $x$ and, for an $f$ which satisfies this condition uniformly on a set $E$ and is bounded on $E$, the convergence is uniform.

Received by the editors October 7, 1998

1991 Mathematics Subject Classification. Primary 26A45, 42A16, 42A20.

Key words and phrases. Bounded variation, Fourier series. 
We use a convergence test of Waterman $[\mathrm{W}]$. For odd integers $n$, let

$$
\begin{aligned}
T_{n}(x, t)= & \frac{f(x+t / n)-f(x+(t+\pi) / n)}{1} \\
& +\frac{f(x+(t+2 \pi) / n)-f(x+(t+3 \pi) / n)}{3} \\
& +\cdots+\frac{f(x+(t+(n-1) \pi) / n)-f(x+(t+n \pi) / n)}{n}
\end{aligned}
$$

and let $Q_{n}(x, t)$ be obtained from $T_{n}(x, t)$ by substituting $-t$ and $-\pi$ for $t$ and $\pi$, respectively.

Convergence Test (Waterman). If $f \in L^{1}(T)$ satisfies the symmetric Lebesgue condition $(*)$ and also satisfies

$$
\int_{\pi}^{2 \pi}\left|T_{n}(x, t)+Q_{n}(x, t)\right| d t=o(1) \quad \text { as } n \rightarrow \infty,
$$

then $S[f, x]$ converges to $f(x)$. If $(*)$ and $(* *)$ hold uniformly on a set $E$ and $f$ is bounded on $E$, then $S[f, x]$ converges uniformly to $f(x)$ on $E$.

If $f \in B V M$, then for a positive integer $k$, and $\Sigma^{o}$ indicating summation over odd integers,

$$
\begin{aligned}
\int_{\pi}^{2 \pi}\left|T_{n}(x, t)\right| d t & =\int_{\pi}^{2 \pi}\left|\sum_{i=1}^{n} o \frac{f(x+(t+(i-1)) \pi / n)-f(x+(t+i \pi / n))}{i}\right| d t \\
& \leq \int\left|\sum_{i=1}^{k} \ldots\right| d t+\int\left|\sum_{i=k+1}^{n} o^{o} \cdots\right| d t \\
& \leq k \omega_{1}\left(f, \frac{\pi}{n}\right)+\frac{1}{k+1} V_{m}(f) .
\end{aligned}
$$

The first term is $o(1)$ as $n \rightarrow \infty$ for fixed $k$ and the second can be made as small as we wish by choosing $k$ large. The corresponding integral with $Q_{n}$ is estimated in the same manner. The test then yields convergence of $S[f, x]$. Note that $(* *)$ holds uniformly on $T$. If $f$ is bounded on $E$ and the Lebesgue condition holds uniformly on $E$, the test yields uniform convergence on $E$. Note that if $f$ is uniformly continuous on $E$, then the Lebesgue condition holds uniformly.

The similarities of the implications for the convergence of $S[f]$ of the property $B V M$ and the property $B V$ lead us to ask, "What functions are in $B V M \backslash B V$ ?".

It is easy to see that even exceptionally regular $f$ which are not of bounded variation may not be in $B V M$. Let $V(f, I)$ denote the variation of $f$ on the interval $I$. Suppose $f$ is a continuous function which is in $C^{1}(a, 2 \pi]$ for every $a \in(0,2 \pi)$ but $V(f,[a, 2 \pi]) \nearrow \infty$ as $a \searrow 0$. Given a partition of $[0,2 \pi]$, let

$$
K_{x}=\left\{k: I_{k x}(\bmod 2 \pi) \subseteq[a, 2 \pi]\right\} .
$$

Then

$$
\sum_{k=1}^{n}\left|f\left(I_{k x}\right)\right| \geq \sum_{k \in K_{x}}\left|f\left(I_{k x}\right)\right|=\sum_{k \in K_{x}}\left|f^{\prime}\left(\theta_{k x}\right)\right|\left(t_{k}-t_{k-1}\right)
$$


for some $\theta_{k x} \in I_{k x}$. For any given $\varepsilon>0$, there is a $\delta_{\varepsilon}>0$ such that $\sup \left(t_{k}-t_{k-1}\right)<$ $\delta_{\varepsilon}$ implies

$$
\sum_{k \in K_{x}}\left|f^{\prime}\left(\theta_{k x}\right)\left(t_{k}-t_{k-1}\right)\right| \geq \int_{a}^{2 \pi}\left|f^{\prime}(t)\right| d t-\varepsilon=V(f,[a, 2 \pi])-\varepsilon .
$$

Thus, for sufficiently fine partitions, we have

$$
\frac{1}{2 \pi} \int_{T} \sum_{k=1}^{n}\left|f\left(I_{k x}\right)\right| d x \geq V(f,[a, 2 \pi])-\varepsilon,
$$

implying that $f \notin B V M$.

This observation leads us naturally to conjecture that $B V M$ does not constitute a true extension of $B V$. The following result shows that this is indeed the case.

Theorem. A function $f \in B V M$ if and only if there is a function $g \in B V$ such that $f=g$ a.e.

Proof. Consider the integral means of a function $f \in L^{1}$,

$$
f_{h}(x)=\frac{1}{h} \int_{0}^{h} f(x+t) d t, \quad h>0 .
$$

Note that these means are absolutely continuous and $f_{h}(x) \rightarrow f(x)$ a.e. as $h \searrow 0$. We have also

$$
f_{h}^{\prime}=\frac{1}{h}(f(x+h)-f(x)) \quad \text { a.e. }
$$

and, therefore,

$$
V\left(f_{h}, T\right)=\frac{1}{h} \int_{T}|f(x+h)-f(x)| d x .
$$

If $f \in B V M$, then there is a $C<\infty$ such that

$$
(* * *) \quad \int_{T} \sum_{i=0}^{n-1}\left|f\left(x+\frac{2 \pi i}{n}\right)-f\left(x+\frac{2 \pi(i+1)}{n}\right)\right| d x<C .
$$

We note that

$$
\begin{aligned}
\int_{T}\left|f\left(x+\frac{2 \pi i}{n}\right)-f\left(x+\frac{2 \pi(i+1)}{n}\right)\right| d x & =\int_{T}\left|f(x)-f\left(x+\frac{2 \pi}{n}\right)\right| d x \\
& =\frac{2 \pi}{n} V\left(f_{2 \pi / n}, T\right) .
\end{aligned}
$$

Thus $(* * *)$ implies that

$$
2 \pi V\left(f_{2 \pi / n}, T\right)<C<\infty,
$$

or $\left\{f_{2 \pi / n}\right\}$ is of uniformly bounded variation. Choose $x_{0}$ such that $f\left(x_{0}\right)$ is finite and $f_{h}\left(x_{0}\right) \rightarrow f\left(x_{0}\right)$ as $h \rightarrow 0$. Then for a given $\varepsilon>0$ there is a $\delta>0$ such that

$$
\left|f_{h}\left(x_{0}\right)-f\left(x_{0}\right)\right|<\varepsilon \text { if } 0<h<\delta
$$

and so

$$
\left|f_{h}\left(x_{0}\right)\right|<\left|f\left(x_{0}\right)\right|+\varepsilon
$$


for such $h$. Thus the sequence $\left\{f_{2 \pi / n}\left(x_{0}\right)\right\}$ is bounded. By Helly's theorem we may deduce the existence of an increasing sequence of the positive integers $\left\{n_{k}\right\}$ and a function $g \in B V$ such that

$$
f_{2 \pi / n_{k}}(x) \rightarrow g(x)
$$

for every $x$ as $k \nearrow \infty$. However

$$
f_{2 \pi / n_{k}}(x) \rightarrow f(x) \quad \text { a.e. }
$$

and so

$$
f(x)=g(x) \in B V \quad \text { a.e. }
$$

\section{REFERENCES}

[MS] Móricz, F., Siddiqi, A. H., A quantified version of the Dirichlet-Jordan test in $L^{1}$-norm, Rend. Circ. Mat. Palermo (2) 45 (1996), no. 1, 19-24. MR 97k:42009

[W] Waterman, Daniel, A generalization of the Salem test, Proc. Amer. Math. Soc. 105 (1989), no. 1, 129-133. MR 89e:42007

Department of Mathematical Sciences, The College of Wooster, Wooster, Ohio 44691

E-mail address: ppierce@acs.wooster.edu

(D. Waterman) Department of Mathematics, Syracuse University, Syracuse, New York 13244

(D. Waterman) Department of Mathematics, Florida Atlantic University, Boca RaTON, FLORIDA 33431

Current address: 7739 Majestic Palm Dr., Boynton Beach, Florida 33437

E-mail address: fourier@earthlink.net 